\title{
Cyberdream: An Interactive Rave Music Visualization in Virtual Reality
}

\author{
Jonathan Weinel \\ School of Computing and \\ Mathematical Sciences \\ University of Greenwich \\ London, UK \\ J.R.Weinel@greenwich.ac.uk
}
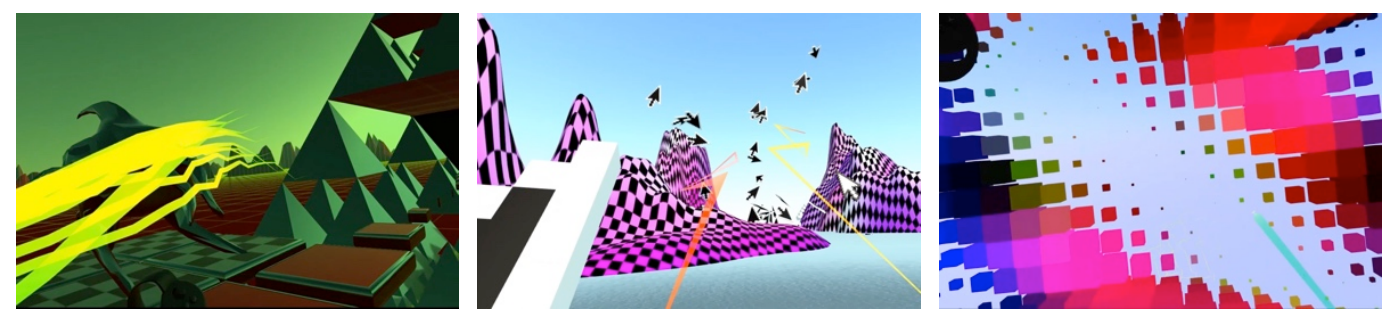

Figure 1: Cyberdream visualizes music in virtual reality, allowing the user to paint with sound using 'StreamerToy' (Left), 'ZigZagToy' (Centre), and 'CircleToy' (Right).

\begin{abstract}
Virtual reality (VR) provides new opportunities for the design of interactive music visualizations. Exploring this area, Cyberdream is a prototype VR application realized through the author's practice-led research, which provides a journey through audiovisual environments based on the aesthetics of 1990s rave music. The project provides three audio-visual 'sound toys', which allow the user to interactively 'paint with sound', thereby facilitating creative play. Through its structural form and audio-visual sound toys, Cyberdream indicates new approaches for the design of music visualizations that harness the spatial properties of VR.
\end{abstract}

\section{CCS CONCEPTS}

- Computing methodologies $\rightarrow$ Computer graphics $\rightarrow$ Graphics systems and interfaces $\rightarrow$ Virtual reality

\section{KEYWORDS}

Music visualization, Virtual reality, Music, Sound design

ACM Reference format:

Jonathan Weinel. 2021. Cyberdream: Interactive Music Visualizations in Virtual Reality. In Proceedings of ACM SIGGRAPH Asia '21. ACM. https://doi.org/[TBA]

\section{Introduction}

From generative visualizations of audio signals, to audio-visual compositions, video jockey (VJ) performances, and visual music films, there are many types of music visualization. Current virtual reality (VR) technologies provide new opportunities to translate these artistic forms into new spatial audio-visual contexts. Expanding the body of work in this area through practice-led research, Cyberdream is a prototype software application, which provides an interactive visualization of 1990sstyle rave music in virtual reality. Extending an earlier version for Oculus GearVR with more limited interactivity [Weinel 2019], the latest iteration of Cyberdream now provides a journey through symbolic audio-visual environments based on 1990s rave culture, in which the user can interactively 'paint with sound' using three audio-visual sound toys. The project aims to demonstrate new compositional methodologies that will advance work in this area.

\section{Context}

In his discussion of 'music in the holodeck', Fischman [2011] proposes the use of immersive audio-visual technologies to provide accessible sonic performance environments that will allow users to interactively and collaboratively compose with sound. These designs can be informed by previous works that visualize sound and music. Early work in this area includes paintings by artists such as Kandinsky, and the visual music animations of Oskar Fischinger, John Whitney and Jordan Belson [Brougher and Mattis 2005]. In the 1990s music visualizers such as Jeff Minter's Virtual Light Machine (VLM) were devised, which created geometric patterns that moved to music [Bell 2019]. At this time VJ performance also emerged as a form in which artists mix live videos to accompany electronic dance music performances [Faulkner 2006].

More recently, we have seen various applications that use game engine technologies and VR to provide audio-visual music experiences. For example, MelodyVR, uses $360^{\circ}$ video cameras to 
provide VR concert experiences; Fantasynth is a non-interactive voyage through an audio-reactive world; and Beat Saber, is a rhythm game where the user swipes at multicolor blocks in time with the beat [Weinel 2020a]. Post COVID-19 there have also been virtual music festivals such as Tomorrowland 2020, where DJs perform in live-streamed computer-generated environments made in game engines such as the Unreal Development Kit. Whilst these works show some possibilities for visualising music in VR, there has been a call for more software applications demonstrating other new compositional approaches and techniques [Turchet, Hamilton and Çamci 2021]. Cyberdream responds to this call by exploring how VJ practices can be translated into the interactive spatial modalities of VR.

\section{Cyberdream}

\subsection{Audio-visual environments}

The audio-visual structure of Cyberdream is based around a journey through spatial environments related to the sounds and images of 1990s rave music. As discussed in Weinel [2020b], these designs were conceived using a symbolic approach, whereby 3D environments are based on the visual language that is associated with the given style of music. In this case, 1990s breakbeat hardcore music [Weinel 2018, 86-87] is visualized by recreating the surrealistic retro-futuristic $3 \mathrm{D}$ landscapes seen in 1990s VJ performances, rave fliers, and record sleeves. As shown in Figure 2, the user flies through a series of audio-visual environments, each of which has a complementary electronic music track, composed for this project using the music tracker Renoise and Roland Cloud. As the user traverses the environments, the music mixes (cross-fades) between the tracks in synchronization in the style of a DJ mix. This is technically accomplished with the game audio middleware software Wwise. In this way the macro structural form of Cyberdream is analogous to a DJ or VJ mix, situating the user inside 3D audiovisual environments through the use of VR.

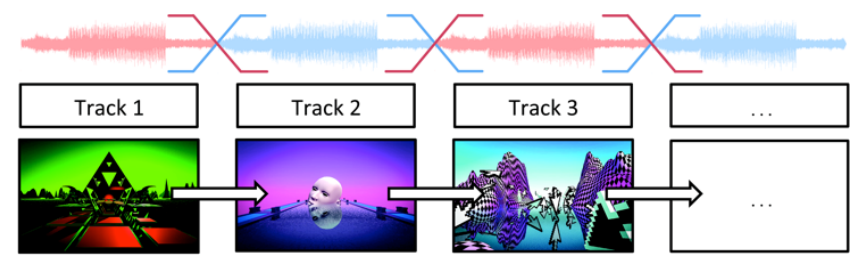

Figure 2: The macro structural form of Cyberdream is analogous to a DJ or VJ mix.

\subsection{Audio-visual sound toys}

The latest version of Cyberdream also provides three audiovisual sound toys (Figure 1), which allow the user to creatively perform with sound and image and 'paint with sound' as they move through the audio-visual environments described. Each toy can be selected and manipulated with the Oculus Touch controllers.
1. 'ZigZagToy' generates jagged orange beams, which emit pulsing sounds and change shape with each pulse. Through the use of callbacks in the Wwise audio engine, the pulses are synchronized precisely with the music, and the user can switch between various rhythmic patterns stored in binary sequences.

2. 'CircleToy' generates rotating blue-green beams, which emit asynchronous pulsing sounds. The user can change the rate and sound of the pulses, which are spatially located based on the direction the user points the beams.

3. 'StreamerToy' emits yellow lightning patterns, which follow gestural movements of the controllers as they fall away from the user. The lightning patterns are accompanied by atonal droning sounds, which are spatially emitted from the tips of the lightning arcs.

All three sound toys provided utilize continuous gestural input via the Oculus Touch controllers, thereby allowing the user to locate the sound and images they produce in 3D space. The sound and visual appearance of beams emitted from all three audio-visual sound toys can also be manipulated with the analogue stick of the Oculus Touch controller, which applies various filters and effects.

\section{Summary}

Cyberdream is a prototype VR application that demonstrates possible strategies for visualizing music, whereby audio-visual environments are designed based on the sounds and visual language of 1990 s rave music. The compositional novelty of the piece arises from the use of scenes analogous to records mixed by a DJ, where each has a corresponding visual and the music crossfades between each in synchronization. Three audio-visual sound toys then allow the user to playfully 'paint with sound', within the complementary framework that the prototype provides, where gestural movements allow the continuous spatial location of sounds and images.

\section{REFERENCES}

Bell, E. 2019. Hacking Jeff Minter's Virtual Light Machine: Unpacking the Code and Community Behind an Early Software-Based Music Visualizer, Volume! 16(1). doi: 10.4000 /volume.7254

Brougher, K. and Mattis, O. 2005. Visual Music: Synaesthesia in Art and Music Since 1900. London: Thames \& Hudson.

Faulkner, M. (D-Fuse) 2006. VJ: Audio-Visual Art and Vj Culture. London: Laurence King.

Fischman, R. 2011. Back to the Parlour, Sonic Ideas/Ideas Sónicas, 3(2): 53-66.

Turchet, L., Hamilton, R., and Çamci, A. 2021. Music in Extended Realities, IEEE Access, 9. doi: 10.1109/ACCESS.2021.3052931

Weinel, J. 2018. Inner Sound: Altered States of Consciousness in Electronic Music and Audio-Visual Media. New York: Oxford University Press.

Weinel, J. 2019. Cyberdream VR: Visualizing Rave Music and Vaporwave in Virtual Reality, ACM Audio Mostly 2019, University of Nottingham, UK. doi: $10.1145 / 3356590.3356637$

Weinel, J. 2020a. Cyberdreams: Visualising Music in Extended Reality (XR). In: Earnshaw, R.A., Liggett, S., Excell, P.S., Thalmann, D. (eds) Technology, Design and the Arts - Challenges and Opportunities. Cham: Springer. doi: 10.1007/9783-030-42097-0_12

Weinel, J. 2020b. Visualising Rave Music in Virtual Reality: Symbolic and Interactive Approaches, EVA London 2020 (Electronic Visualisation and the Arts). doi: $10.14236 /$ ewic/EVA2020.13 